\title{
Article
}

\section{Impact of the Application of Computer-Based 3D Simulation on Acquisition of Knowledge of Guidance of Mandibular Movement}

\author{
Hai Yen Mai ${ }^{1}$, Hang-Nga Mai ${ }^{2}$, Hyun-Wook Woo ${ }^{1}$ and Du-Hyeong Lee ${ }^{1,2, *(D)}$ \\ 1 Department of Prosthodontics, School of Dentistry, Kyungpook National University, Daegu 41940, Korea; \\ maihaiyen@knu.ac.kr (H.Y.M.); itrd@knu.ac.kr (H.-W.W.) \\ 2 Institute for Translational Research in Dentistry, Kyungpook National University, Daegu 41940, Korea; \\ maihangnga1403@knu.ac.kr \\ * Correspondence: deweylee@knu.ac.kr; Tel.: +82-53-600-7676
}

Citation: Mai, H.Y.; Mai, H.-N.; Woo, H.-W.; Lee, D.-H. Impact of the Application of Computer-Based 3D Simulation on Acquisition of Knowledge of Guidance of Mandibular Movement. Appl. Sci. 2021, 11, 60. https://dx.doi. org/10.3390/app11010060

Received: 8 December 2020

Accepted: 21 December 2020

Published: 23 December 2020

Publisher's Note: MDPI stays neutral with regard to jurisdictional claims in published maps and institutional affiliations.

Copyright: (c) 2020 by the authors. Licensee MDPI, Basel, Switzerland. This article is an open access article distributed under the terms and conditions of the Creative Commons Attribution (CC BY) license (https: / / creativecommons.org/ licenses/by/4.0/).
Abstract: Recently, computer-aided three-dimensional (3D) simulation has expanded to modern education. This study aims to investigate the effects of 3D computer simulation on the learning and self-assessment of the guidance of the mandibular movement. Sixty second-grade dental students were randomly distributed into three groups in an occlusion class. Various teaching protocols were used for each group. Students in the first group (lecture (L)) were taught exclusively through a textbook and two-dimensional illustrations. The conventional lecture method followed by computeraided 3D simulation was applied to the second group (lecture-to-simulation (LtS)). Lastly, students in the third group (lecture with simulation $(\mathrm{LwS})$ ) were simultaneously taught using the conventional lecture and computer-aided 3D simulation methods. After teaching each group, a paper-based examination was conducted; actual and expected scores were obtained on the same day as the occlusal class. Analyses of variance with Tukey's post-hoc analysis were used to compare the teaching protocols, whereas the independent $t$ test was used for comparing between actual and expected scores $(\alpha=0.05)$. The LwS group exhibited significantly higher actual and student-expected scores than the L and LtS groups $(p<0.001)$. The expected score was significantly lower than the actual score in the L group $(p=0.035)$. However, in the LtS and LwS groups, no statistical difference was observed between expected $(p=0.114)$ and actual $(p=0.685)$ scores. The distribution of actual scores in the grading systems indicated higher percentages of excellent (grade A) and good (grade B) scores in the LwS (96.7\%) and LtS (79.7\%) groups, respectively, than in the L group (53.4\%). Using computer-aided 3D simulation to teach the guidance of mandibular movement improved the learning outcomes and self-assessment of students, especially when 3D simulation was combined with conventional lecturing.

Keywords: dental occlusion; simulation; visualization; computer software; learning effect; selfassessment; guidance; mandibular movement

\section{Introduction}

With recent technological breakthroughs, computer-assisted three-dimensional (3D) simulation has been widely applied to training courses for education in various medical fields. Three-dimensional simulation provides a virtual environment similar to the clinical setting [1]. The current literature reports that the effect of computer simulation on knowledge acquisition is equal or superior to that of traditional teaching methods [2], as evidenced by improvements in the critical thinking and spatial perception of students and clinical correlations. Today, methods for computer-based 3D simulation have been extended to dental education, specifically in training courses for diagnosis in periodontology and orthodontics, caries removal, tooth preparation, and extraction $[3,4]$.

Understanding the guidance of mandibular movement is essential for the accurate diagnosis of occlusal diseases and treatment for prosthetic rehabilitation [5]. The movement of the mandible is mainly related to two anatomic structures, namely, the teeth and 
temporomandibular joints [6]. The contact surfaces between the maxillary and mandibular anterior teeth determine anterior guidance, whereas the shape of the articular eminence of the temporal bone influences posterior guidance. Vertical and horizontal overlap of the anterior teeth is required to disengage the posterior teeth during excursive movements of the mandible and prevent harmful lateral forces on the posterior teeth. The specific trace and orientation of the mandible are set via the interaction between the teeth, joint structure, and neuromuscular system. Knowledge of related anatomic structures and understanding of the controlling factors in anterior and posterior guidance are important because the functional movement of the mandible influences chewing efficiency, phonetics and esthetics.

Student learning outcomes are directly dependent on the relationship between the nature of the knowledge to be conveyed and the educational method used [7]. Interpretation of the complex spatial relationships and kinetics of mandibular movement can be challenging [8]. Illustrating the complex movement of the mandible and the dynamic nature of dental occlusion is difficult when using conventional teaching methods such as textbooks and two-dimensional images. Such crucial, yet complicated, knowledge is made more understandable through the use of 3D graphic visualization and simulation [9]. Previous research [10] on learner responses and learning effects related to the principles of occlusal adjustment compared the use of paper-based 2D presentations and computer-based 3D simulation methods, and concluded that 3D computer imaging helps learners acquire the required knowledge of occlusal adjustment.

The purpose of this study was to assess the effects of computer-assisted 3D simulation on learning and self-assessment related to the guidance of mandibular movement. To conduct the evaluation, three teaching protocols, namely, lecture (L), lecture-to-simulation $(\mathrm{LtS})$, and lecture with simulation $(\mathrm{LwS})$, were compared with regard to actual and studentexpected learning outcomes. The null hypothesis is that integrating computer-aided simulation into the teaching protocol on dental occlusion does not influence learning outcomes.

\section{Materials and Methods}

Figure 1 provides a summary of the workflow of the study, which was approved by the ethics research board of Kyungpook National University (KNUDH-2020-03-06). The study was conducted on 60 dental students, wherein recruitment was limited to second-year dental undergraduate students from the school of dentistry of one university. All the students followed the same curriculum. This inclusion criterion was applied to control for confounding factors that may influence the results, such as background of dental knowledge and practice experiences. The power analysis method was used to determine the number of participants per group (effect size $f=0.597, \alpha=0.05$, power $=0.8$, standard deviation $=10.3$, number of groups $=3$ ) [10]. All participants were blinded to the objective of the study and randomly assigned into three groups $(n=20)$ using the adaptive pair-matching method based on grade point average [11].

The three abovementioned protocols were individually used to teach the students, under the L, LtS, and LwS groups, respectively, the guidance of the mandibular movement. Conventional lecture methods using a textbook and 2D illustrations were employed to teach the L group (Figure 2) [12]. Students in the LtS group were taught with the conventional lecture method followed by computer-aided 3D simulation using a computer design software program (R2CAD; MegaGen, Daegu, Korea) to simulate the movements of the mandible (Figure 3). Meanwhile, students in the LwS group were simultaneously taught using the conventional lecture and computer-aided 3D simulation methods. An experienced instructor delivered the lesson content for all groups. 


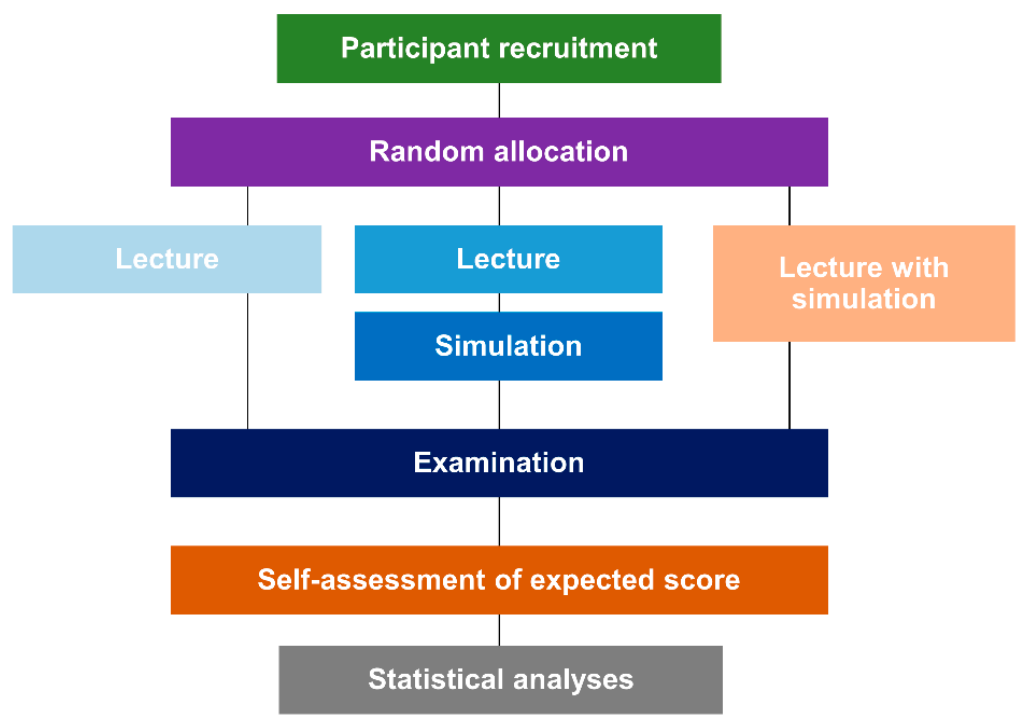

Figure 1. Workflow for this study.
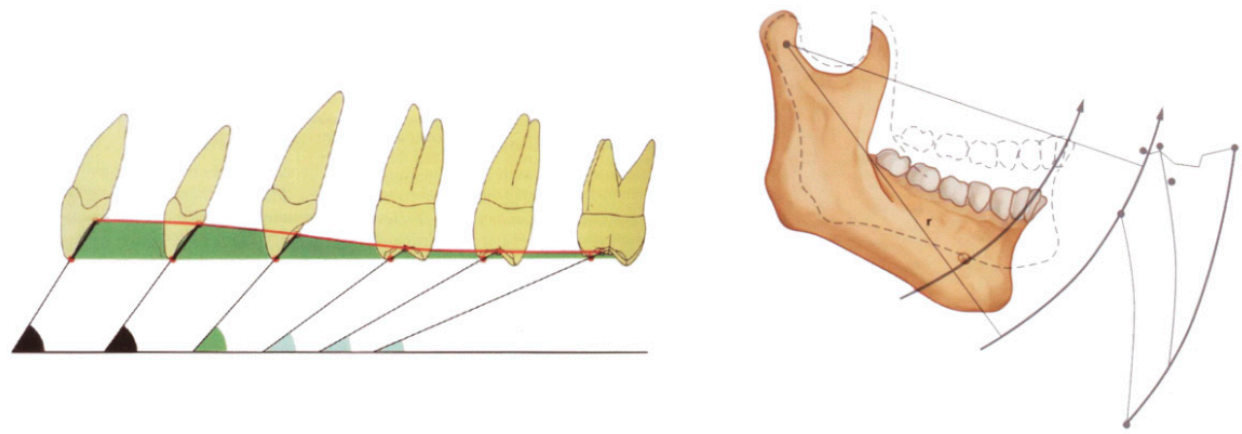

Figure 2. Lecture-based teaching method with two-dimensional schematic illustrations.
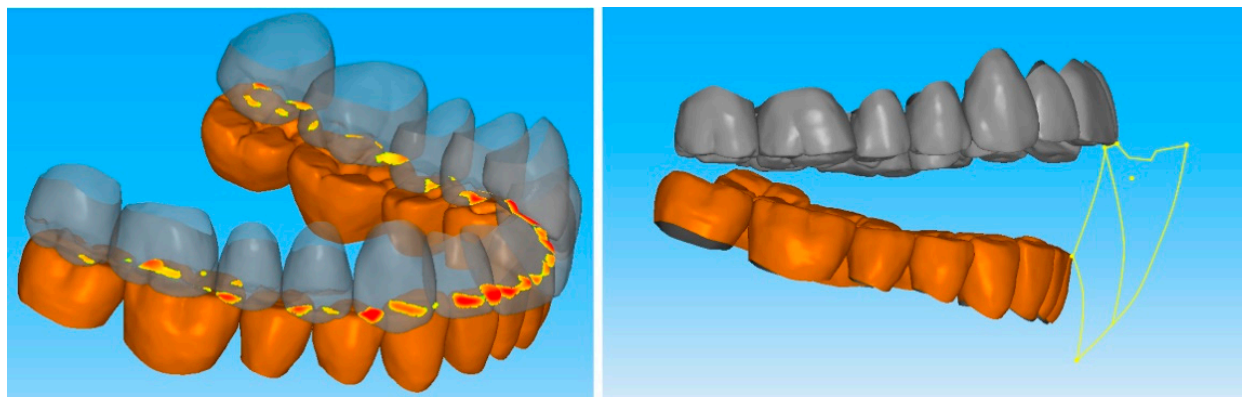

Figure 3. Computer visualization and simulation method with three-dimensional models.

To assess learning outcomes, a paper-based examination (Supplementary Materials S1) was conducted on the day of the lesson. The examination comprised nine closed-ended questions [13] that aimed to investigate the understanding of the students regarding the anterior and posterior determinants of occlusion and mandibular movement. Students were given scores on a scale of $0-100$. The numerical scores were then converted to letter grades as follows: $\mathrm{A}=>90$ to $\leq 100, \mathrm{~B}=>80$ to $\leq 90, \mathrm{C}=>70$ to $\leq 80, \mathrm{D}=>60$ to $\leq 70$, and $\mathrm{F}=\leq 60$, with $\mathrm{A}$ being the highest and $\mathrm{F}$ being the lowest grade [11]. Scoring was conducted twice; first, by the students to constitute expected grades and second by the course instructor as actual grades. Upon completion of data collection and to adhere to research ethics protocols, later in the semester, students in the lecture group were taught using the 3D simulation method, and students in the 3D simulation group were taught using the lecture method. 
The means and standard deviations of the actual and expected scores were calculated. Analysis of variance with Tukey's post-hoc analysis was used for comparing between teaching protocols, whereas the independent $t$ test was used for the actual and expected scores. All statistical analyses were performed using a statistical software program (IBM SPSS Statistics, v25.0; IBM Corp, Armonk, NY, USA). A $p$ value of 0.05 was considered significant.

\section{Results}

Table 1 provides the results between the actual and expected scores. The mean actual score of the LwS group was significantly higher than those of the L and LtS groups $(p<0.001$; Figure 4). No statistical difference was observed between the results of the $\mathrm{L}$ and LtS groups. In term of expected scores, the L group exhibited significantly lower estimates than the other groups that used the computer-aided simulation

Table 1. Mean and standard deviation of examination scores for groups following different teaching protocols.

\begin{tabular}{ccccc}
\hline Score & Lecture & Lecture to Simulation & Lecture with Simulation & $p^{*}$ \\
\hline Actual & $82.7 \pm 8.6^{\mathrm{a}}$ & $85.2 \pm 7.4^{\mathrm{a}}$ & $90.7 \pm 7.1^{\mathrm{b}}$ & $<0.001$ \\
Expected & $75.7 \pm 16.9^{\mathrm{a}}$ & $88.7 \pm 10.7^{\mathrm{b}}$ & $89.7 \pm 12.5^{\mathrm{b}}$ & $<0.001$ \\
$p^{+}$ & 0.035 & 0.114 & 0.685 & \\
\hline
\end{tabular}

* Statistical differences within rows were calculated using one-way analysis of variance with Tukey's post-hoc analysis. Same superscript lowercase letters show no significant differences among 3 groups within row. ${ }^{+}$Statistical differences within columns were calculated using the independent $t$ test.

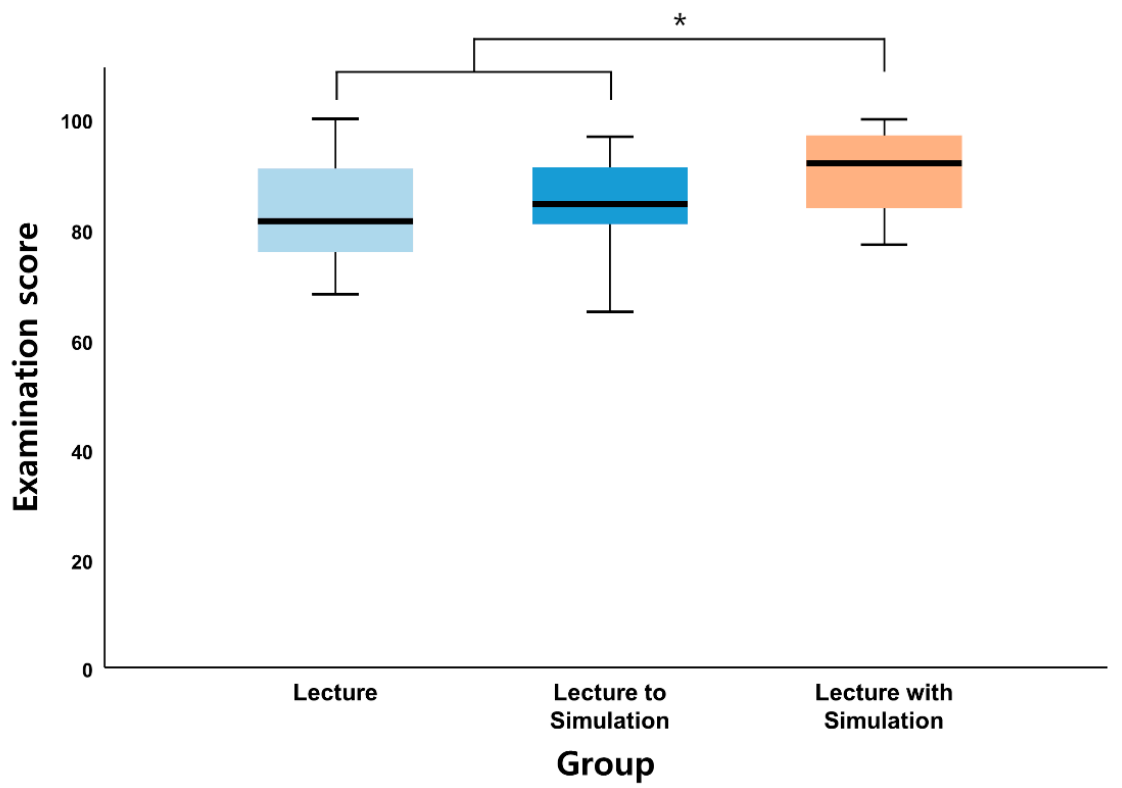

Figure 4. Box-plot diagram for examination scores according to the different protocols for occlusal education. * Statistical differences.

Between the actual and expected scores, the mean expected score was significantly lower than the mean actual score for the L group $(p=0.035)$. However, no statistical difference was found between the actual $(p=0.685)$ and expected $(p=0.114)$ scores for the $\mathrm{LtS}$ and LwS groups.

The grading system illustrates the distribution of the actual scores for each group (Figure 5). In general, high percentages of excellent (grade A) and good (grade B) scores were observed with the application of computer-aided simulation in teaching compared with the sole use of the lecture method. The highest recorded score intervals for the LwS and L groups were grades A and C, respectively. For all teaching protocols, a failure score of grade $\mathrm{F}$ was not observed. 


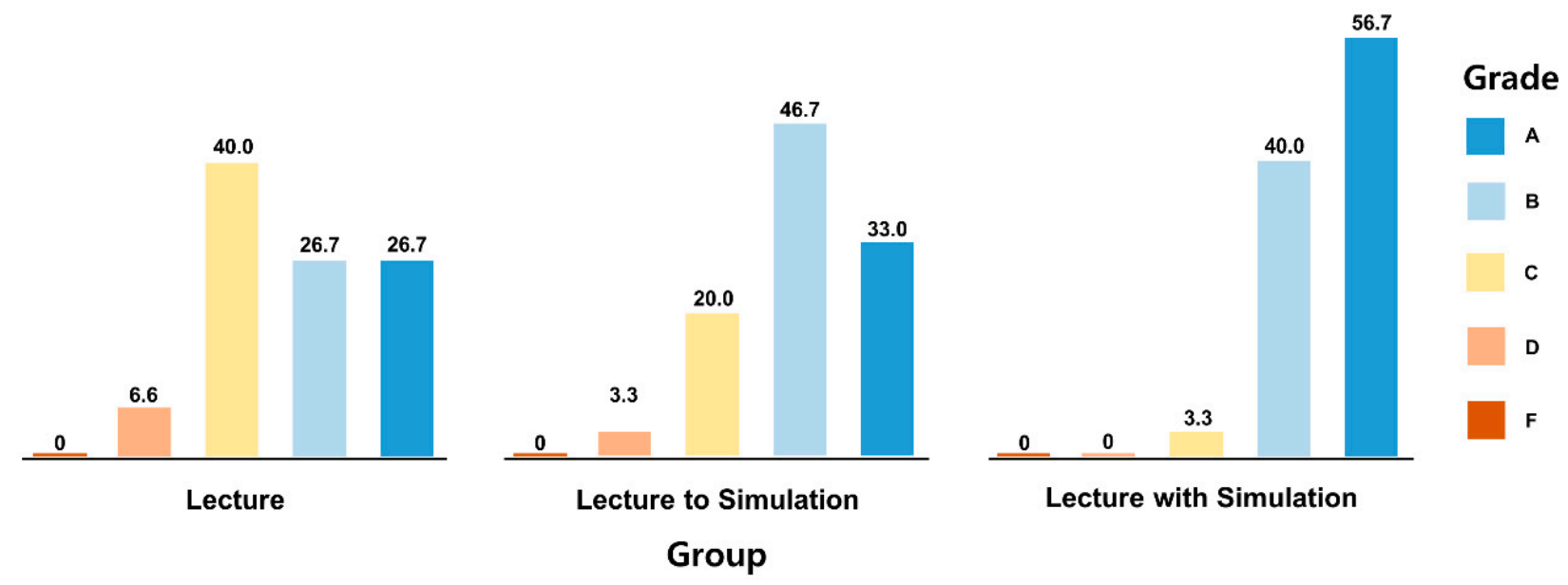

Figure 5. Grading of examination scores.

\section{Discussion}

The study aimed to evaluate the effects of computer-aided simulation on the learning outcomes of students in terms of mandibular movement. The results showed significant improvements when 3D simulation was simultaneously used with the conventional lecture method as a teaching protocol for the determinants of guidance and mandibular movement. Thus, the null hypothesis that including computer-aided simulation in the teaching strategy for occlusion would not influence learning outcomes was rejected.

The study compared the actual and expected examination scores to assess the influence of the teaching methods on learning outcomes. The actual score reflected knowledge acquisition, whereas the expected score indicated the students' self-confidence in and expectation regarding learning [14]. The results indicated that students who received the lecture-only-based teaching protocol displayed relatively low estimates of their grade outcomes, which implies that they were unsure about their understanding of the presented knowledge. As such, they may have experienced difficulty in being confident about their level of knowledge acquisition when the complicated spatial relationships of dentition were explained through text and 2D illustration. A learner's confidence in their learning knowledge influences the human cognitive processes related to learning and memory, reasoning, and problem-solving abilities [15]. Previous studies demonstrated that negative expectations and emotions can significantly decrease memorization [16,17]. Meanwhile, including computer-aid simulation in the teaching protocol for mandibular movement increased their expectation about knowledge acquisition. In 3D visualization, the use of computer software and jaw mesh models enabled the reproduction of the setting for guidance and mandibular movement in a virtual space. In this way, students could intuitively identify the relationship between tooth and condylar guidance, and the path of mandibular movement. Not only academic achievement but also confidence in knowledge acquisition was considered to increase through the application of computer-aided visualization.

In this study, a significant difference was observed in the academic achievement of students in different groups despite the same 3D simulation being applied to the LtS and $\mathrm{LwS}$ groups. The simultaneous application of 3D simulation with conventional lecturing methods led to higher examination scores than when both methods were applied separately. This reaffirms the importance of the timing and arrangement of teaching methods for their educational impact. The simultaneous application of 3D simulation is considered acceptable for dental occlusion teaching, given the dynamic nature of the neuromuscular system in the oral cavity and the diversified functional movements of the mandible. Further experimental studies incorporating a variety of time factors are required.

The authors are unaware of previous research using 3D computer image software to simulate mandibular movement for teaching occlusion. Although one study [10] investigated the effects of computer-based 3D simulation on teaching occlusion, the 3D simulation 
was applied to teach occlusal adjustments in centric and eccentric positions in the mandible, and the 3D simulation protocol was not subdivided. The findings of the current study extend the application of computer modeling and design for understanding the spatial movement of the mandible.

Lecture-based teaching using textbooks and 2D illustration remains mainstream in dental education. This approach is suitable for conveying core knowledge and concepts, especially to a large audience while maintaining the consistency and quality of education at relatively low costs [18]. However, despite the benefits of lecture-based education, previous research demonstrated that lectures were inefficient with regard to the teaching skills and application of facts or critical reasoning [19,20] required for certain courses, such as clinical medicine and dentistry. As such, understanding the 3D structural and functional elements of human anatomy remains challenging [21,22]. Conventional tools for visualization, such as 2D illustrations and schematics, are prone to error in the conceptualization of such knowledge, where wrongly entrenched knowledge disturbs the further acquisition of related knowledge [23,24]. Recent advances in imaging technologies have enabled the 3D simulation of the dynamics of anatomic structures and interactive learning, thus facilitating the understanding of complex phenomena $[25,26]$. Furthermore, various fields of dentistry $[4,27]$ have documented the advantages of using 3D models.

Certain influencing limitations should be considered in interpreting the results of the current study. First, only one type of commercial computer software was used to apply 3D visualization. Therefore, further studies should investigate the effects of computer software by including diverse programs that utilize different features and parameters in controlling and handling images. Second, this study did not evaluate inter- and intra-learner reliability in terms of learning effects. With the involvement of multiple learners, the analysis of variability among learners could be important. Third, this study is limited by its sample size. Although expanding the number of participants was difficult due to the need to control for background knowledge and related previous experiences, a larger sample is required to generalize the study findings. Therefore, comprehensive multi-center studies are required to confirm the results of this study.

\section{Conclusions}

Despite the limitations, the study found that incorporating computer-aided 3D simulation into the teaching protocol for teaching the guidance of mandibular movement improved the actual and expected learning outcomes of the students. Moreover, the simultaneous application of 3D simulation and the conventional lecture method rendered better effects on learning compared to when both were used as separate approaches for teaching.

Supplementary Materials: The following is available online at https:/ / www.mdpi.com/2076-3417/ 11/1/60/s1, S1: Examination on the determinants in the dental occlusion.

Author Contributions: Conceptualization, H.Y.M. and D.-H.L.; methodology, H.Y.M. and D.-H.L.; data curation, H.Y.M., H.-N.M., H.-W.W. and D.-H.L.; formal analysis, H.Y.M. and D.-H.L.; investigation, H.Y.M., H.-N.M., H.-W.W. and D.-H.L.; writing-original draft preparation, H.Y.M., H.-N.M. and H.-W.W.; writing-review and editing, D.-H.L.; supervision, D.-H.L. All authors have read and agreed to the published version of the manuscript.

Funding: This research received no external funding.

Institutional Review Board Statement: The study was conducted according to the guidelines of the Declaration of Helsinki, and ap-proved by the Institutional Review Board (or Ethics Committee) of Kyungpook National Uni-versity Dental Hospital (KNUDH-2019-08-01-00).

Informed Consent Statement: Participant compliance was waived because the data of this study was obtained within regular educational curriculum.

Data Availability Statement: Data available on request due to restrictions on privacy (student's test score).

Conflicts of Interest: The authors declare no conflict of interest. 


\section{References}

1. De Boer, I.R.; Wesselink, P.R.; Vervoorn, J.M. The creation of virtual teeth with and without tooth pathology for a virtual learning environment in dental education. Eur. J. Dent. Educ. 2013, 17, 191-197. [CrossRef] [PubMed]

2. Jwayyed, S.; Stiffler, K.A.; Wilber, S.T.; Southern, A.; Weigand, J.; Bare, R.; Gerson, L.W. Technology-assisted education in graduate medical education: A review of the literature. Int. J. Emerg. Med. 2011, 4, 51. [CrossRef] [PubMed]

3. Urbankova, A. Impact of computerized dental simulation training on preclinical operative dentistry examination scores. J. Dent. Educ. 2010, 74, 402-409. [CrossRef] [PubMed]

4. Pohlenz, P.; Gröbe, A.; Petersik, A.; von Sternberg, N.; Pflesser, B.; Pommert, A.; Höhne, K.-H.; Tiede, U.; Springer, I.; Heiland, M. Virtual dental surgery as a new educational tool in dental school. J. Craniomaxillofac. Surg. 2010, 38, 560-564. [CrossRef] [PubMed]

5. Clark, J.R.; Evans, R.D. Functional occlusion: I. A review. J. Orthod. 2001, 28, 76-81. [CrossRef]

6. McCullock, A.J. Making occlusion work: I. Terminology, occlusal assessment and recording. Dent. Update 2003, 30, 150-157. [CrossRef]

7. O'Carroll, E.; Leung, A.; Fine, P.D.; Boniface, D.; Louca, C. The teaching of occlusion in undergraduate dental schools in the UK and Ireland. Br. Dent. J. 2019, 227, 512-517. [CrossRef]

8. Park, S.; Kim, Y.; Park, S.; Shin, J.-A. The impacts of three-dimensional anatomical atlas on learning anatomy. Anat. Cell Biol. 2019, 52, 76-81. [CrossRef]

9. Bando, E.; Nishigawa, K.; Nakano, M.; Takeuchi, H.; Shigemoto, S.; Okura, K.; Satsuma, T.; Yamamoto, T. Current status of researches on jaw movement and occlusion for clinical application. Jpn. Dent. Sci. Rev. 2009, 45, 83-97. [CrossRef]

10. Mai, H.Y.; Mai, H.-N.; Lee, D.-H. Computer-Based 3D Simulation Method in Dental Occlusion Education: Student Response and Learning Effect. Appl. Sci. 2020, 10, 6703. [CrossRef]

11. Anderson, L. A critique of grading: Policies, practices, and technical matters. Educ. Policy Anal. Arch. 2018, 26, 49. [CrossRef]

12. Gnanasegaram, J.J.; Leung, R.; Beyea, J.A. Evaluating the effectiveness of learning ear anatomy using holographic models. J. Otolaryngol. Head Neck Surg. 2020, 49, 63. [CrossRef] [PubMed]

13. Husain, H.; Bais, B.; Hussain, A.; Samad, S.A. How to Construct Open Ended Questions. Procedia Soc. Behav. Sci. 2012, 60, 456-462. [CrossRef]

14. Doménech-Betoret, F.; Abellán-Roselló, L.; Gómez-Artiga, A. Self-Efficacy, Satisfaction, and Academic Achievement: The Mediator Role of Students' Expectancy-Value Beliefs. Front. Psychol. 2017, 8, 1193. [CrossRef]

15. Tyng, C.M.; Amin, H.U.; Saad, M.N.M.; Malik, A.S. The Influences of Emotion on Learning and Memory. Front. Psychol. 2017, 8, 1454. [CrossRef]

16. Pekrun, R. The Impact of Emotions on Learning and Achievement: Towards a Theory of Cognitive/Motivational Mediators. Appl. Psychol. 1992, 41, 359-376. [CrossRef]

17. Seli, P.; Wammes, J.D.; Risko, E.F.; Smilek, D. On the relation between motivation and retention in educational contexts: The role of intentional and unintentional mind wandering. Psychon. Bull. Rev. 2016, 23, 1280-1287. [CrossRef]

18. Cantillon, P. Teaching large groups. BMJ 2003, 326, 437. [CrossRef]

19. Wiens, J. Fundamentals of Occlusion, 1st ed.; American College of Prosthodontists: Chicago, IL, USA, 2015.

20. Sobierajewicz, J.; Szarkiewicz, S.; Przekoracka-Krawczyk, A.; Jaśkowski, W.; van der Lubbe, R. To What Extent Can Motor Imagery Replace Motor Execution While Learning a Fine Motor Skill? Adv. Cogn. Psychol. 2016, 12, 179-192. [CrossRef]

21. Naidoo, N.; Akhras, A.; Banerjee, Y. Confronting the Challenges of Anatomy Education in a Competency-Based Medical Curriculum During Normal and Unprecedented Times (COVID-19 Pandemic): Pedagogical Framework Development and Implementation. JMIR Med. Educ. 2020, 6, e21701. [CrossRef]

22. Pujol, S.; Baldwin, M.; Nassiri, J.; Kikinis, R.; Shaffer, K. Using 3D Modeling Techniques to Enhance Teaching of Difficult Anatomical Concepts. Acad. Radiol. 2016, 23, 507-516. [CrossRef] [PubMed]

23. Estai, M.; Bunt, S. Best teaching practices in anatomy education: A critical review. Ann. Anat. 2016, 208, 151-157. [CrossRef] [PubMed]

24. Singh, K.; Bharatha, A.; Sa, B.; Adams, O.P.; Majumder, M.A.A. Teaching anatomy using an active and engaging learning strategy. BMC Med. Educ. 2019, 19, 149. [CrossRef] [PubMed]

25. Frizziero, L.; Santi, G.M.; Liverani, A.; Napolitano, F.; Papaleo, P.; Maredi, E.; di gennaro, G.; Zarantonello, P.; Stallone, S.; Stilli, S.; et al. Computer-Aided Surgical Simulation for Correcting Complex Limb Deformitiesin Children. Appl. Sci. 2020, 10, 5181. [CrossRef]

26. Kwon, C. The Effect of the Degree of Anxiety of Learners during the Use of VR on the Flow and Learning Effect. Appl. Sci. 2020, 10, 4932. [CrossRef]

27. Liu, L.; Li, J.; Yuan, S.; Wang, T.; Chu, F.; Lu, X.; Hu, J.; Wang, C.; Yan, B.; Wang, L. Evaluating the effectiveness of a preclinical practice of tooth preparation using digital training system: A randomized controlled trial. Eur. J. Dent. Educ. 2018, 22, e679-e686. [CrossRef] 